\title{
Resonance energy transfer based on shallow and deep energy levels of biotin-polyethylene glycol/polyamine stabilized CdS quantum dots
}

\author{
W. Lü ${ }^{\text {a) }}$ \\ Quantum Nanotechnology Laboratory, Konan University, Kobe 658-8501, Japan \\ Y. Tokuhiro \\ Department of Physics, Konan University, Kobe 658-8501, Japan \\ I. Umezu and A. Sugimura \\ Quantum Nanotechnology Laboratory, Konan University, Kobe 658-8501, Japan \\ and Department of Physics, Konan University, Kobe 658-8501, Japan \\ Y. Nagasaki \\ Tsukuba Research Center for Interdisciplinary Material Science, University of Tsukuba, \\ Tsukuba 305-8573, Japan
}

(Received 21 July 2006; accepted 24 August 2006; published online 6 October 2006)

\begin{abstract}
Fluorescent resonance energy transfer between the poly(ethylene glycol)- $b$-poly(2( $N, N$-dimethylamino)ethyl methacrylate) stabilized $\mathrm{CdS}$ quantum dots (QDs) and texas-red streptavidin was observed. We propose a four-state model to explain photoluminescence (PL) process of CdS QDs and suggest that there are two emission processes originated from shallow and deep trap energy levels corresponding to fast and slow components of PL decay, respectively. Energy transfer mechanism was discussed based on Dexter theory [J. Chem. Phys. 21, 863 (1953)] and the proposed four-state model. It is found that the energy transfer efficiency of deep energy level is higher than that of shallow energy level. The calculated distance between QD and texas red with the parameters of shallow energy level is the same with that of deep level, which indicates that the proposed model is reasonable for explaining the PL dynamics of CdS QDs. (C) 2006 American Institute of Physics. [DOI: 10.1063/1.2359295]
\end{abstract}

Semiconductor nanocrystals represent a potential class of labeling probes because of their size dependent optical properties, exceptional photochemical stability, broad excitation spectra, and narrow emission bands ${ }^{1}$ and have been intensively studied in biological applications. ${ }^{2-5}$ However, the dispersion stability of semiconductor quantum dots (QDs) in aqueous media decreases with decreasing size in the range of nanometers due to increased surface area. In this regard, polymer-supported stabilization of the QDs is one of the effective methods. ${ }^{6-8}$ In this work, poly(ethylene glycol)$b$-poly (2-( $N, N$-dimethylamino)ethyl methacrylate) (PEG/ PAMA) molecule was used as a polymer to stabilize the surface of QDs. The biotin-PEG/PAMA stabilized QDs as energy donors in conjunction with dye acceptors were prepared. The dispersion stability in aqueous media was greatly improved. Fluorescent resonance energy transfer (FRET) was observed by the specific interaction between biotinPEG/PAMA stabilized CdS QDs and texas-red-labeled streptavidin. $^{9}$ Although PEG/PAMA improved the dispersion stability in aqueous media, it has a disadvantage for FRET since PEG/PAMA chains have larger length than those of molecules generally used to stabilize QDs. ${ }^{10}$ Therefore it is important to investigate the energy transfer mechanism of the present system and, further, to improve FRET efficiency and to estimate QD-dye distance. In order to clarify the FRET quantitatively, we studied time-resolved photoluminescence (PL) spectra. A four-state model is proposed to explain PL

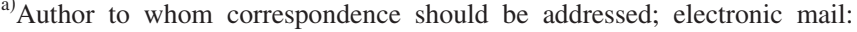
luwei@center.konan-u.ac.jp
}

dynamics of CdS QDs, and energy transfer mechanism is discussed based on Dexter theory.

The PEG/PAMA and biotin-PEG/PAMA block copolymer are synthesized by previously reported method. ${ }^{9,12}$ To an $8 \mathrm{ml}$ aqueous solution of the block copolymer (3.08 $\times 10^{-4} \mathrm{~mol} / \mathrm{l}$ as amine concentration in the block copolymer) in a glass vial, $\mathrm{CdCl}_{2}\left(2.5 \times 10^{-3} \mathrm{~mol} / \mathrm{l}\right)$ and $\mathrm{Na}_{2} \mathrm{~S}$ $\left(2.5 \times 10^{-3} \mathrm{~mol} / \mathrm{l}\right)$ were added in this order and stirred for $1 \mathrm{~h}$ at room temperature. The purification was carried out by dialysis against water. The biotin-installed CdS QDs was prepared in a similar manner using the biotin-PEG/PAMA block copolymer. The PEG/PAMA and biotin-PEG/PAMA stabilized CdS QDs are named as PEG-CdS and biotin-PEG$\mathrm{CdS}$ in this letter, respectively. Texas red streptavidin was added to biotin-PEG-CdS to observe FRET from CdS QDs to texas red. This sample is named texas-red-PEG-CdS. PL spectrum and time-resolved PL intensity were measured by a monochromator with gated charge coupled device camera. Excitation source was third harmonics of neodymium doped yttrium aluminum garnet laser $(355 \mathrm{~nm})$. The pulse width of the laser was about $6 \mathrm{~ns}$.

PL and absorption spectra of PEG-CdS are shown in Fig. 1. The absorption peak of PEG-CdS at $3.5 \mathrm{eV}$ indicates that the mean diameter of the QDs characterized by quantum confinement model is about $4 \mathrm{~nm} .{ }^{13}$ Comparing absorption spectrum and PL spectrum of CdS QDs, it can be found that the PL peak energy of PEG-CdS is much lower than absorption peak energy. This indicates the PL peak does not come from intrinsic band gap, which is attributed to the recombination of trapped charge carriers. ${ }^{14}$ The PL spectrum changes with the delay time of measurement after the excitation 


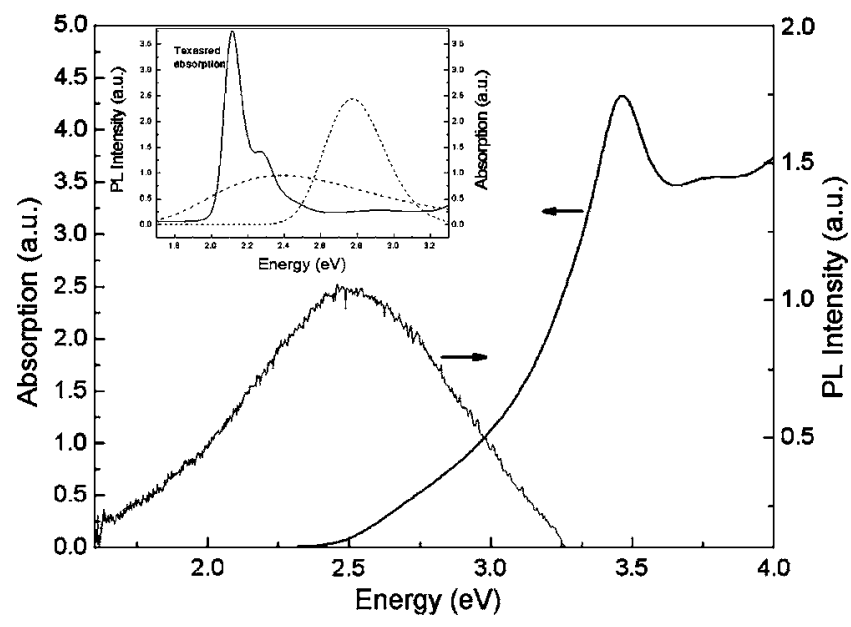

FIG. 1. PL and absorption spectra of PEG-CdS. Inset: the normalized PL spectra of shallow and deep level emissions and the normalized absorption spectrum of texas red.

pulse, as shown in Fig. 2. The inset shows variation of the photon energy corresponding to the peak intensity with delay time. The peak energy decreases from 2.72 to $2.41 \mathrm{eV}$ with increasing delay time up to $100 \mathrm{~ns}$ and after that it becomes constant. Time-resolved PL intensity of PEG-CdS shown in Fig. 3 also exhibits two components. Based on these results as well as previously reported result that the lifetime of shallow energy level is shorter than that of deep energy level, ${ }^{15}$ we suggest that the observed PL peak is composed of fast shallow and slow deep components. Due to the fast decay of PL intensity of the shallow energy level emission, the intensity ratio of shallow level emission to that of deep level in PL band decreases with delay time and the PL peak moves toward lower energy edge (deep energy level). The shallow trap level is attributed to surface defect states and the deep trap level possibly originated from the impurities substituted in the sulfur sites. ${ }^{16}$ The result of convolution of biexponential decay function with the instrumental response is fitted to the experimental data, as shown in Fig. 3. The time constants are obtained by this fitting. For shallow level, the time constants of PEG-CdS and texas red combined PEG-CdS are 6 and $4.5 \mathrm{~ns}$; for deep level, the time constants are 70 and $27 \mathrm{~ns}$, respectively. In order to find the peak position of shal-

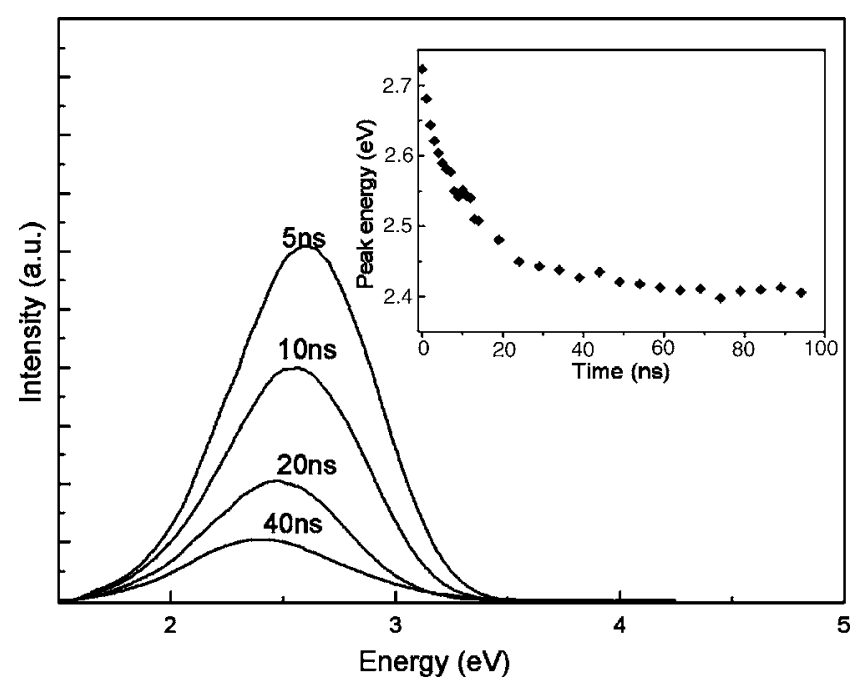

FIG. 2. PL peaks of PEG-CdS at different delay times. Inset: peak position change with the increasing gate delay time.

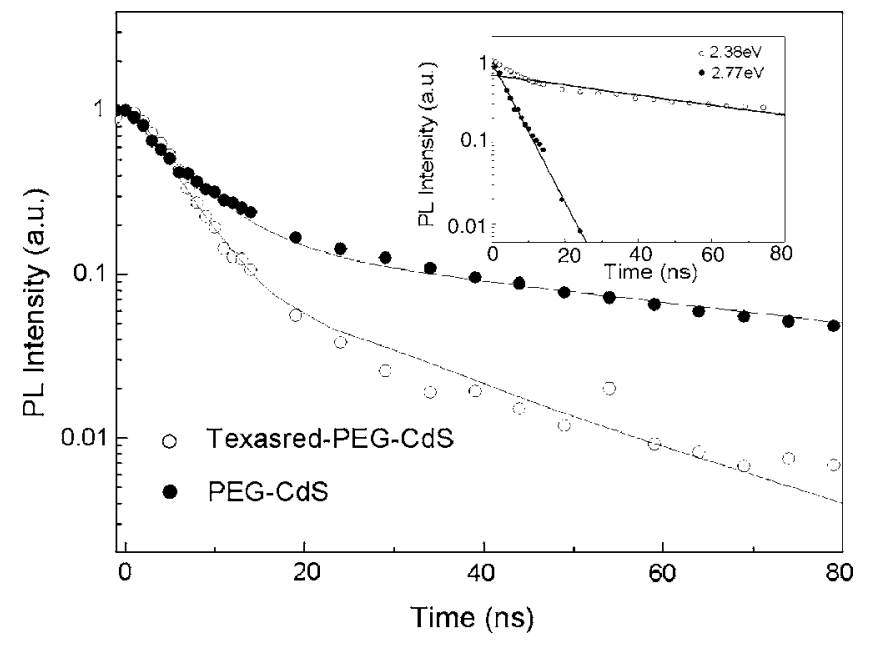

FIG. 3. Time-resolved PL intensities of PEG-CdS and texas-red-PEG-CdS. Inset: the time-resolved PL intensities of decomposed peaks at 2.77 and $2.38 \mathrm{eV}$ for PEG-CdS. The solid lines are fitting curves for experimental data.

low and deep energy levels, PL peaks of PEG-CdS at different delay times are decomposed to two peaks at two different energy positions; the lower energy peak corresponds to deep energy level and the higher energy peak corresponds to shallow energy level. The time constants of the two decomposed peaks are compared with the time constants acquired from time-dependent PL intensity. Decomposition results show that the time constants from decomposition at 2.77 and $2.38 \mathrm{eV}$ are consistent with result of time-dependent PL intensity. The time-resolved PL intensities of the two peaks are shown in inset of Fig. 3. This result further proves that two trap states dominate emission process. The PL lifetime of PEG-CdS decreases after addition of texas-red streptavidin, as shown in Fig. 3, which directly justifies the existence of FRET from CdS QDs to texas red.

Here, we propose a four-state model to explain our experimental results and to investigate FRET efficiency of the present system by Dexter theory. ${ }^{11}$ Figure 4 is the proposed four-state model. For PEG-CdS, $P$ is excitation to absorption energy level $S_{1}$ from ground state. There are a shallow trap state $S_{2}$ and a deep trap state $S_{3}$ under absorption energy level $S_{1}$. The electrons at $S_{1}$ decay to $S_{2}$ and to $S_{3}$ through nonradiative process with probabilities $\gamma_{1}$ and $\gamma_{1}^{\prime}$, respectively. Then electrons at $S_{2}$ and $S_{3}$ recombine to ground state with probabilities $\gamma_{2}$ and $\gamma_{3}$, respectively. The excited state $S_{2}$ is proposed to relax to the ground state in a radiative process without passing through state $S_{3}$. After addition of texas-red streptavidin, due to dipole-dipole interaction, some electrons at $S_{1}$ and $S_{2}$ are transferred to excited state $A$ of

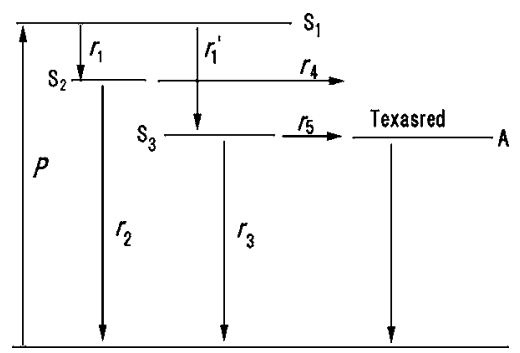

FIG. 4. Proposed four-state model for explaining PL dynamics of PEG-CdS and texas-red-PEG-CdS. 
texas red with probabilities $\gamma_{4}$ and $\gamma_{5}$ in nonradiative process. FRET rate and efficiency can be expressed as

$$
\begin{aligned}
& \gamma_{d d}=\gamma_{C+T}-\gamma_{C}=\frac{1}{\tau_{C+T}}-\frac{1}{\tau_{C}}, \\
& \eta=\frac{\gamma_{d d}}{\gamma_{d d}+\gamma_{c}}=1-\frac{\tau_{C+T}}{\tau_{C}},
\end{aligned}
$$

where $\eta$ is FRET efficiency, $\gamma_{d d}$ is FRET rate, $\gamma_{C}$ is decay probability of PEG-CdS, $\gamma_{C+T}$ is decay probability of texasred-PEG-CdS, and $\tau_{C}$ and $\tau_{C+T}$ are time constants of PEG-CdS and texas-red-PEG-CdS, respectively. For shallow energy level, $\tau_{C}$ is $1 / \gamma_{2}$ and $\tau_{C+T}$ is $1 /\left(\gamma_{2}+\gamma_{4}\right)$; for deep energy level, $\tau_{C}$ is $1 / \gamma_{3}$ and $\tau_{C+T}$ is $1 /\left(\gamma_{3}+\gamma_{5}\right)$. The energy transfer rates for shallow and deep levels are 0.06 and $0.023 \mathrm{~ns}^{-1}$, respectively. The calculated FRET efficiency of deep energy level $S_{3}(62 \%)$ is higher than that of shallow energy level $S_{2}(27 \%)$.

According to Dexter, ${ }^{11}$ the transfer rate for dipole-dipole FRET process from a particular sensitizer to a particular activator is expressed as follows

$$
\gamma_{d d}=\frac{3 \hbar^{4} c^{4} Q_{a}}{4 \pi R^{6} n^{4} \tau_{s}}\left(\frac{\varepsilon}{\kappa^{1 / 2} \varepsilon_{c}}\right)^{4} \int \frac{f_{s}(E) F_{a}(E)}{E^{4}} d E,
$$

where $n$ is refractive index of medium, $\tau_{s}$ is the decay constant of sensitizer and $\left(\varepsilon_{c} / \varepsilon\right)^{2}=1 / \kappa, Q_{a}$ is the area under absorption band of activator, $R$ is distance between sensitizer and activator, and $\int\left\{\left[f_{s}(E) F_{a}(E)\right] / E^{4}\right\} d E$ is the spectral overlap integral between normalized sensitizer emission and normalized activator absorption line shapes. The emission of PEG-CdS is dominated by shallow and deep levels and the overlap integrals from shallow and deep level emissions are different. The steady-state PL spectrum in Fig. 1 can be decomposed into two peaks corresponding to shallow and deep levels at 2.77 and $2.38 \mathrm{eV}$. The emission peaks of the two levels are shown in inset of Fig. 1. The distance between CdS QD (sensitizer) and texas red (activator) can be calculated with different overlap integrals and time constants of shallow and deep levels, respectively. Following parameters are used in calculation: $n=1.3318, \hbar=1.05459 \times 10^{-27} \mathrm{erg} \mathrm{s}$, $Q_{a}=6.9018 \times 10^{-17} \mathrm{~cm}^{2} \mathrm{eV}$, and the overlap integral $\int\left\{\left[f_{s}(E) F_{a}(E)\right] / E^{4}\right\} d E$ can be estimated from inset of Fig. 1. The distances calculated using the decay constants of shallow energy level and deep energy level are 4.9 and $4.7 \mathrm{~nm}$, respectively. The error comes from the definition of trap level position because different positions will induce to dif- ferent overlap integral values. The small difference between the two calculated distances manifests our model is reasonable for explaining the PL dynamics of CdS QDs. It is consistent with the values $(1-10 \mathrm{~nm})$ generally reported in the literature for an efficient energy transfer process, too. ${ }^{17}$

In summary, a four-state model is proposed to explain the PL behavior of CdS QDs. FRET from CdS QDs to texas red was observed. Energy transfer mechanism was discussed based on Dexter theory and the proposed four-state model. Time constants acquired from PL peak decomposition and the distances calculated using the parameters of shallow and deep energy levels indicate that the proposed model is reasonable.

The authors are grateful to T. Ishii and Y. Sunaga, Tokyo University of Science, for the preparation of biotin-PEG/ PAMA block copolymers and the polymer modified CdS QDs.

${ }^{1}$ A. P. Alivisatos, Science 271, 933 (1996).

${ }^{2}$ D. Alexson, H. Chen, M. Cho, M. Dutta, Y. Li, P. Shi, A. Raichura, D. Ramadurai, S. Parikh, M. Stroscio, and M. Vasudev, J. Phys.: Condens. Matter 17, R637 (2005).

${ }^{3}$ M. Stroscio and M. Dutta, Proc. IEEE 93, 1772 (2005).

${ }^{4}$ D. Ramadurai, B. Kohanpour, D. Alexson, P. Shi, A. Sethuraman, Y. Li, V. Saini, M. Dutta, and M. Stroscio, Proc. IEEE 151, 189 (2004).

${ }^{5}$ D. Alexson, Y. Li, D. Ramadurai, P. Shi, L. George, L. George, M. Uddin, P. Thomas, S. Rufo, M. Dutta, and M. Stroscio, IEEE Trans. Nanotechnol. 3, 86 (2004).

${ }^{6}$ J. Huang, K. Sooklal, C. J. Murphy, and H. J. Ploehn, Chem. Mater. 11, 3595 (1999).

${ }^{7}$ K. Sooklal, L. H. Hanus, H. J. Ploehn, and C. J. Murphy, Adv. Mater. (Weinheim, Ger.) 10, 1083 (1998).

${ }^{8}$ N. Kumbhojkar, S. Mahamuni, V. Leppert, and S. H. Risbud, Nanostruct. Mater. 10, 117 (1998).

${ }^{9}$ Y. Nagasaki, T. Ishii, Y. Sunaga, Y. Watanabe, H. Otsuka, and K. Kataoka, Langmuir 20, 6396 (2004).

${ }^{10}$ I. Umezu, R. Koizumi, A. Sugimoto, M. Inada, T. Makino, A. Sugimura, Y. Sunaga, T. Ishii, and Y. Nagasaki, Physica E (Amsterdam) 21, 1102 (2004).

${ }^{11}$ D. L. Dexter, J. Chem. Phys. 21, 836 (1953).

${ }^{12}$ K. Kataoka, A. Harada, D. Wakebayashi, and Y. Nagasaki, Macromolecules 32, 6892 (1999).

${ }^{13}$ J. Allegre, G. Arnaud, H. Mathieu, P. Lefebvre, W. Granier, and L. Boudes, J. Cryst. Growth 138, 998 (1994).

${ }^{14}$ M. Dib, M. Chamarro, V. Voliotis, and J. L. Fave, Phys. Status Solidi B 212, 293 (1999).

${ }^{15}$ J.-C. Seo, D. Kim, and H. J. Kong, Appl. Phys. A: Mater. Sci. Process. 64, 445 (1997).

${ }^{16}$ K. Misawa, H. Yao, T. Hayashi, and T. Kobayashi, Chem. Phys. Lett. 183, 113 (1991).

${ }^{17}$ J. R. Lakowicz, Principles of Fluorescence Spectroscopy, 2nd ed. (Plenum, New York, 1999). 